Draft VERSION JANUARY 1, 2021

Typeset using LATEX modern style in AASTeX63

\title{
Timing of Eight Binary Millisecond Pulsars Found with Arecibo in Fermi-LAT Unidentified Sources
}

\author{
J. S. Deneva, ${ }^{1}$ P. S. Ray,${ }^{2}$ F. Camilo,${ }^{3}$ P. C. C. Freire, ${ }^{4}$ H. T. Cromartie,${ }^{5}$ \\ S. M. Ransom,${ }^{6}$ E. Ferrara,${ }^{7}$ M. Kerr,${ }^{2}$ T. H. Burnett,${ }^{8}$ And \\ P. M. SAZ PARKINSON ${ }^{9,10}$ \\ ${ }^{1}$ George Mason University, resident at the Naval Research Laboratory, Washington, DC 20375, USA \\ ${ }^{2}$ Naval Research Laboratory, Washington, DC 20375, USA \\ ${ }^{3}$ South African Radio Astronomy Observatory, Cape Town, 7925, South Africa \\ ${ }^{4}$ Max-Planck-Institut für Radioastronomie, Auf dem Hügel 69, D-53121 Bonn, Germany \\ ${ }^{5}$ University of Virginia, Charlottesville, VA 22904, USA \\ ${ }^{6}$ National Radio Astronomy Observatory, Charlottesville, VA 22903, USA \\ ${ }^{7}$ Goddard Space Flight Center, Greenbelt, MD 20771, USA \\ ${ }^{8}$ University of Washington, Seattle, WA 98195, USA \\ ${ }^{9}$ Department of Physics and Laboratory for Space Research, The University of Hong Kong, \\ Pokfulam Road, Hong Kong \\ ${ }^{10}$ Santa Cruz Institute for Particle Physics, University of California, Santa Cruz, CA, 95064, USA
}

\begin{abstract}
We present timing solutions for eight binary millisecond pulsars (MSPs) discovered by searching unidentified Fermi-LAT source positions with the $327 \mathrm{MHz}$ receiver of the Arecibo 305-m radio telescope. Five of the pulsars are "spiders" with orbital periods shorter than $8.1 \mathrm{~h}$. Three of these are in "black widow" systems (with degenerate companions of $0.02-0.03 \mathrm{M}_{\odot}$ ), one is in a "redback" system (with a non-degenerate companion of $\left.\gtrsim 0.3 \mathrm{M}_{\odot}\right)$, and one $(\mathrm{J} 1908+2105)$ is an apparent middle-ground case between the two observational classes. The remaining three pulsars have white dwarf companions and longer orbital periods. With the initially derived radio timing solutions, we detected $\gamma$-ray pulsations from all MSPs and extended the timing solutions using photons from the full Fermi mission, thus confirming the identification of these MSPs with the Fermi-LAT sources. The radio emission of the redback is eclipsed during $50 \%$ of its orbital period, which is typical for this kind of system. Two of the black widows exhibit radio eclipses lasting for $10-20 \%$ of the orbit, while J1908+2105 eclipses for $40 \%$ of the orbit. We investigate an apparent link between gamma-ray emission and a short orbital period among known binary MSPs in the Galactic disk, and conclude that selection effects cannot be ruled out as the cause. Based on this analysis we outline how the likelihood of new MSP discoveries can be improved in ongoing and future pulsar searches.
\end{abstract}

\section{INTRODUCTION}


The Fermi-LAT Fourth Source Catalog (4FGL, Abdollahi et al. 2020) contains more than 1300 gamma-ray sources not associated with an object type or a counterpart in another energy band. This constitutes $\sim 26 \%$ of all 4FGL sources, which are detected based on eight years of data from the Fermi Large Area Telescope (LAT, Atwood et al. 2009). Since 2008, the first year of the Fermi mission, more than 250 pulsars have been detected in gamma rays (Abdo et al. 2013) ${ }^{1}$, up from $<10$ before Fermi was launched. Many of the pulsars newly detected in gamma rays by Fermi-LAT were discovered in blind radio surveys both before and after Fermi was launched. However, more than half of the newly identified gamma-ray pulsars were found in targeted searches of previously unassociated Fermi-LAT sources. These are broadly divided into two different classes. The first class consists mostly of young pulsars, which tend to be isolated and have rotation periods of tens to hundreds of milliseconds. In this case it is possible to perform a blind search for gamma-ray pulsations using only Fermi-LAT photon data (Abdo et al. 2009). This method has produced more than 60 gamma-ray pulsar discoveries to date (e.g. Saz Parkinson et al. 2010, Pletsch et al. 2012a).

In contrast, millisecond pulsars (MSPs) tend to be in binary systems and have rotation periods of only a few ms. The short period in combination with the unknown orbital parameters make a blind gamma-ray pulsation search extremely computationally intensive and difficult within a reasonable computing time. Nevertheless, the discovery that known MSPs are an important class of gamma-ray emitters (Abdo et al. 2009b) has given a crucial impetus to this difficult search. In some cases the search can become feasible when astrometric and orbital parameters are constrained through optical observations (Pletsch et al. 2012b, Nieder at al. 2020, Clark et al. 2020). An alternative method relies on searches for radio pulsars at the positions of unidentified Fermi sources. These surveys have found more than 80 MSPs ( e.g. Ransom et al. 2011; Cognard et al. 2011; Keith et al. 2011; Ray et al. 2012; Camilo et al. 2015; Cromartie et al. 2016) ; these represent about 25\% of all known MSPs to date in the Galactic disk (hereafter referred to as 'field MSPs' to distinguish them from MSPs residing in globular clusters away from the Galactic plane which are subject to different evolution scenarios and selection biases). After a timing solution is obtained based on 1-2 years of regular radio observations, gamma-ray pulsations are most often detected after Fermi-LAT photon arrival times are binned according to the radio ephemeris. If gamma-ray pulsations are detected, in most cases it is possible to extend the timing solution to $>10$ years by using gamma-ray photons and bootstrapping from the shorter span of the radio ephemeris.

In this paper we present timing solutions and gamma-ray pulsation detections for eight binary MSPs discovered in radio searches of Fermi-LAT unassociated sources. Seven of the solutions span the full Fermi mission. In one case even though gamma-

\footnotetext{
${ }^{1}$ For an up-to-date list, see https://confluence.slac.stanford.edu/display/GLAMCOG/Public+List+of+LAT-Detected-
} 
ray pulsations were detected, it was not possible to extend the timing solution outside the span of radio observations due to rapid quasi-periodic variations in the orbital period.

Section 2 describes our radio observations and the data analysis that produces the initial phase-connected timing solutions. Section 3 describes the analysis of gammaray photon data and how we extend the timing solutions to the full length of the Fermi mission. Section 4 presents the timing solutions for the pulsars; and Section 5 focuses on the different types of MSP binaries represented in this work and their properties and implications.

\section{RADIO OBSERVATIONS AND DATA ANALYSIS}

The MSPs in this paper were found in targeted searches of unassociated Fermi-LAT sources with pulsar-like gamma-ray spectra. Cromartie et al. (2016) announced the discovery of six of the MSPs (J0251+2606, J1805+0615, J1824+1014, J1908+2105, $\mathrm{J} 2052+1219$, and J1048+2339), and later searches identified two more (J1625-0021 and J2006+0148). The search observations used the $327 \mathrm{MHz}$ receiver at the Arecibo telescope. Our subsequent regular timing observations continued with the same setup. We used the Puerto Rican Ultimate Pulsar Processing Instrument (PUPPI), a clone of the GUPPI intrument operating at the Green bank telescope (DuPlain et al. 2008) in incoherent search mode with a bandwidth of $100 \mathrm{MHz}$ and sampling time of $81.92 \mu \mathrm{s}$. Because the backend bandwidth exceeds the receiver bandwidth, samples were recorded to disk only from the $68.75 \mathrm{MHz}$ within the PUPPI band corresponding to the bandwidth of the $327 \mathrm{MHz}$ receiver.

During the first month of timing observations, we dedispersed each data set with the dispersion measure (DM) of the pulsar determined during the discovery to remove the frequency-dependent time delay of the pulse across the bandwidth. Then we summed all channels to produce a one-dimensional time series, and used accelsearch from PRESTO ${ }^{2}$ to perform a Fast Fourier Transform search for periodic signals. This step produces a measurement of the barycentric rotational period $\left(P_{\text {bary }}\right)$ and apparent period derivative, which is related to the line-of-sight acceleration during the observation.

\subsection{Orbital Solutions}

After the accumulation of several closely spaced observations over the span of a month, it was evident that the MSPs are in binary systems as we observed $P_{\text {bary }}$ to vary. This variation is due to Doppler shift induced by orbital motion. We used two methods in succession to obtain an initial fit for the orbital parameters. The first method is described by Freire et al. (2001) and exploits the fact that for a nearly circular orbit (the most common scenario for MSPs), measurement points in the 2D space defined by $P_{\text {bary }}$ and the line-of-sight acceleration fall on an ellipse. This method

\footnotetext{
${ }^{2}$ https://www.cv.nrao.edu/ ${ }^{\text {sransom/presto }}$
} 
yields a rough fit to the orbital parameters even with only a handful of observations, as long as they are spread over at least half the orbit. However, the ellipse fit needs measurements of line-of-sight acceleration within individual observations, which are typically only a few minutes long. For one of our MSPs, PSR J1824+1014, no acceleration was detectable in any of the observations, indicating that the orbital period is on the order of weeks to months. In this case, we used a Lomb-Scargle periodogram on the measurements of $P_{\text {bary }}$ vs. time to obtain an initial estimate of the orbital period. Next we used these initial fits as a starting point for fitting a sinusoid to $P_{\text {bary }}$ vs. time in order to refine our estimate of the orbital parameters.

\subsection{Pulse Times-of-Arrival}

We average ("fold") the data from each observation with the initial ephemeris, which includes $P_{\text {bary }}$, the orbital parameters, and the nominal sky position of the unidentified Fermi-LAT source, which is also the center of the discovery radio beam. While individual pulses may differ in shape and height, the average pulse profile is stable in time if areas with extra dispersive delays near eclipse ingress and/or egress are excluded. We fit a set of Gaussians to the folded pulse profile with the highest signal-to-noise ratio. The result is a noiseless multi-Gaussian pulse template, which is then convolved with the average pulse profiles from non-overlapping stretches of data from each observation in order to extract pulse times-of-arrival (TOAs). For each observation, we initially extract $2-5$ TOAs from each of two frequency subbands, with each pair of TOAs calculated from 5-10 minutes of data. The specific number of TOAs from each observation is determined by the observation length, the brightness of the detection, and whether the observation includes eclipse ingress or egress, which shortens the usable portion of data.

\subsection{Phase-Connected Timing Solutions}

For each pulsar, we use the TOAs in TEMPO ${ }^{3}$ with the DE421 Solar System ephemeris to obtain a least-squares fit to rotational, astrometric, and orbital parameters, as well as the DM by minimizing the sum of the squares of the differences between actual and expected TOAs. In order to do this we need to determine the correct number of pulsar rotations between TOAs; this is generally achieved using the manual method described in detail by Freire \& Ridolfi (2018), section 3. However, five of the pulsars (PSRs J1805+0615, J1824+1014, J1908+2105, J2006+0148 and J2052+1219) are only visible when a part of the Galactic plane is visible at Arecibo. The telescope's high over-subscription rate at those times implies that not much time is available to follow up these pulsars, which results in a relatively sparse data set. In order to determine the correct rotation count between TOAs, we used the "Dracula" script, which is described by Freire \& Ridolfi (2018), section 4. Once the correct rotation counts have been achieved, precise measurements of the rotational, astrometric,

\footnotetext{
${ }^{3}$ http://tempo.sourceforge.net
} 
and orbital parameters can be made. At this point, we freeze the DM in the timing solution. We redo the TOA extraction, obtaining only one TOA per observation. Since these TOAs are calculated over the entire frequency band and a longer time span, they have smaller error bars than the TOAs in the initial set and allow us to refine the ephemeris by using them to refit the timing parameters (except DM) with TEMPO. The residuals of this final set of radio TOAs with respect to the post-fit radio timing solution are shown in Figures 1-2.

The radio timing solutions for our eight pulsars have time spans of $2.4-3.4$ years. However, since all pulsars were found in Fermi-LAT unassociated sources, we can use our radio timing solutions as a starting point to search for gamma-ray pulsations and extend the timing solution span to the entire $>10$ year span of the Fermi mission.

\subsection{Flux Density Estimates}

Along with the timing solution for each pulsar we give an estimate of the flux density at $327 \mathrm{MHz}$. For each observation we calculate the uncalibrated period-averaged flux density based on the radiometer equation, the pulse profile template used to extract the TOAs, and using nominal values for the $327 \mathrm{MHz}$ receiver system temperature $\left(T_{\text {sys }}=113 \mathrm{~K}\right)$ and gain $(G=11 \mathrm{~K} / \mathrm{Jy})$. The recorded value is the average across all observations, excluding non-detections and observations taken near eclipse where the pulsar is not detected during the full integration time (e.g. Figure 5).

\section{FERMI-LAT DATA ANALYSIS}

We searched for gamma-ray pulsations from each pulsar using Pass 8 Fermi-LAT data (Atwood et al. 2013, Bruel et al. 2018) from 2008 August 4 to 2019 July 31. We extracted events identified as "Source" class (evclass $=128$, evtype $=3$ ) and detected during periods flagged as producing good science data (DATA_QUAL = 1 , LAT_CONFIG = 1). Additional event cuts were based on zenith angle $\left(<90^{\circ}\right)$ and energy $(0.1-100 \mathrm{GeV})$. We extracted events satisfying these criteria within $15^{\circ}$ of the pulsar's timing position. Then we performed a binned likelihood analysis of a $20^{\circ} \times 20^{\circ}$ region centered on the same position. The sky is modeled using the 4FGL catalog and its corresponding background gll_iem_v07 and iso_P8R3_SOURCE_V2_v1.txt. We fit the spectrum normalization parameters for the two background components.

If the spectrum of the Fermi-LAT source corresponding to the pulsar was originally modeled with a power law, we modified it to a power law with an exponential cut-off,

$$
\frac{d N}{d E}=N_{0} \frac{E^{-\Gamma}}{E_{0}} e^{-E / E_{c}},
$$

where $\Gamma$ is the power law index, $E_{c}$ is the cut-off energy, and $N_{0} / E_{0}$ is a normalization constant. For our maximum likelihood fit we used the P8R3_SOURCE_V2 instrument response functions and Science Tools version 11-00-07. We fit the spectral parameters of the pulsar source and the normalization parameter of variable sources within $6^{\circ}$ of it. All other parameters were kept fixed at their 4FGL catalog values. Next we 
used the obtained spectral model to assign weights to photons within $2^{\circ}$ of the pulsar according to the procedure described by Kerr (2011).

We calculated the photon phases based on the radio timing solution using PINT. ${ }^{4}$ Since the time span of Fermi-LAT data for all our pulsars except J1048+2339 is $>10$ years, much longer than the $2-3$ year span of the radio timing solutions, the gamma-ray pulse profile drifts in phase outside the range of the radio ephemerides, where the radio timing parameters do not optimally predict the pulsar rotation. We used event_optimize from PINT (Luo et al. 2020) to obtain 11-year timing solutions using the radio ephemerides as a starting point and applying a Markov Chain Monte Carlo technique for fitting timing model parameters (Foreman-Mackey et al. 2013). Based on the H-test significance values of the gamma-ray pulsations, we find the best results when optimizing the position, rotation period, period derivative, and orbital parameters including the orbital period, projected semi-major axis, and time of passage through periastron or ascending node. MCMC-optimized parameters in Tables 1-3 are reported as maximum likelihood values with upper and lower uncertainties corresponding to the bounds of the $68 \%$ confidence interval for each parameter.

The MCMC-optimized timing positions of all eight MSPs are within the 95\% confidence contours of their respective 4FGL sources. All positions except that of J1625-0021 are also within the 4FGL 68\% confidence contours.

\section{RESULTS}

\subsection{PSR J0251+2606}

This eclipsing black widow MSP was discovered in the Fermi-LAT source 3FGL J0251.1+2603 (4FGL J0251.0+2605). The radio timing residuals vs. observing epoch and orbital phase are shown in Figures 1 and 2. The Fermi timing solution is shown in Table 2. The pulsar has an orbital period of 4.86 hours and a minimum companion mass of $0.025 \mathrm{M}_{\odot}$. Using the $\sim 3$-yr radio timing solution as a starting point and optimizing it based on Fermi-LAT photon data from the full 11-year gamma-ray data set, we were able to detect gamma-ray pulsations with a H-test significance of $383.08(18 \sigma)$. Figure 3 (top left) shows the photon phaseogram and folded gamma-ray profile phase-aligned with the $327 \mathrm{MHz}$ radio profile. The alignment is done using PINT to calculate photon phases with respect to the same phase reference used for extracting radio TOAs and to construct an average gamma-ray pulse profile.

\subsection{PSR J1625-0021}

This MSP was discovered in the Fermi-LAT source 3FGL J1625.1-0021 (4FGL J1625.1-0020). The radio timing residuals vs. observing epoch are shown in Figure 1. The Fermi timing solution is shown in Table 1. The pulsar has an orbital period of 7.4 days and a minimum companion mass of $0.17 \mathrm{M}_{\odot}$. The high

\footnotetext{
${ }^{4}$ https://github.com/nanograv/PINT
} 
Table 1. Parameters for three pulsar binary systems with likely helium white dwarf companions. All ephemerides use Barycentric Dynamical Time (TDB) units. Uncertainties in parentheses are $1 \sigma$ errors from TEMPO fitting. Parameters reported with \pm uncertainties are maximum likelihood values from MCMC optimization. In those cases the uncertainties correspond to the $68 \%$ confidence interval bounds. The pulsar distance is estimated based on the DM and the YMW16 model of Galactic ionized gas density (Yao et al. 2017).

\begin{tabular}{|c|c|c|c|}
\hline \multicolumn{4}{|c|}{ Observation and data-set parameters } \\
\hline Pulsar name ................................ & $\mathrm{J} 1625-0021$ & J1824+1014 & J2006+0148 \\
\hline MJD range $\ldots \ldots \ldots \ldots \ldots \ldots \ldots \ldots \ldots \ldots \ldots \ldots$ & $54682.7-58696.0$ & $54682.7-58696.0$ & $54682.7-58696.0$ \\
\hline 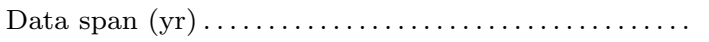 & 11.0 & 11.0 & 11.0 \\
\hline Reference epoch (MJD) ..................... & 57114 & 56925 & 57243 \\
\hline \multicolumn{4}{|c|}{ Measured Quantities } \\
\hline Right ascension, $\alpha$ (hh:mm:ss) $\ldots \ldots \ldots \ldots \ldots \ldots \ldots$ & $16: 25: 10.3579_{-0.0003}^{+0.0002}$ & $18: 24: 14.933_{-0.003}^{+0.001}$ & $20: 06: 29.0529_{-0.0001}^{+0.0002}$ \\
\hline Declination, $\delta$ (dd:mm:ss) $\ldots \ldots \ldots \ldots \ldots \ldots \ldots$ & $-00: 21: 28.960_{-0.008}^{+0.008}$ & $+10: 14: 43.82_{-0.03}^{+0.02}$ & $+01: 48: 53.919_{-0.006}^{+0.007}$ \\
\hline Dispersion measure, $\mathrm{DM}\left(\mathrm{cm}^{-3} \mathrm{pc}\right) \ldots \ldots \ldots \ldots \ldots$ & 16.497 & 59.8807 & 43.5786 \\
\hline Pulse frequency, $\nu\left(\mathrm{s}^{-1}\right) \ldots \ldots \ldots \ldots \ldots \ldots \ldots$ & $352.90623328676_{-3 \times 10^{-11}}^{+2 \times 10^{-11}}$ & $245.9470303117_{-1 \times 10^{-10}}^{+3 \times 10^{-10}}$ & $462.20187192375_{-3 \times 10^{-11}}^{+3 \times 10^{-11}}$ \\
\hline First derivative of pulse frequency, $\dot{\nu}\left(10^{-15} \mathrm{~Hz} \mathrm{~s}^{-1}\right)$. & $-2.6559_{-0.0005}^{+0.0004}$ & $-0.3247_{-0.003}^{+0.0009}$ & $-0.703_{-0.003}^{+0.008}$ \\
\hline Proper motion in right ascension, $\mu_{\alpha} \cos \delta\left(\operatorname{mas~yr}^{-1}\right)$ & $-3.6(13)$ & - & - \\
\hline Proper motion in declination, $\mu_{\delta}\left(\right.$ mas $\left._{\mathrm{yr}^{-1}}\right) \ldots \ldots \ldots$ & $-8.9(42)$ & - & - \\
\hline Binary model $\ldots \ldots \ldots \ldots \ldots \ldots \ldots \ldots \ldots \ldots \ldots$ & ELL1 & DD & ELL1 \\
\hline Orbital period, $P_{\mathrm{b}}(\mathrm{d}) \ldots \ldots \ldots \ldots \ldots \ldots \ldots \ldots \ldots \ldots \ldots \ldots \ldots$ & $7.38297056_{-1 \times 10^{-8}}^{+1 \times 10^{-8}}$ & $82.548980_{-1 \times 10^{-6}}^{+2 \times 10^{-6}}$ & $0.6513597615_{-6 \times 10^{-10}}^{+4 \times 10^{-10}}$ \\
\hline Projected semi-major axis of pulsar's orbit, $x$ (lt-s).. & $4.584723_{-8 \times 10^{-6}}^{+6 \times 10^{-6}}$ & $35.44585_{-4 \times 10^{-5}}^{+3 \times 10^{-5}}$ & $0.842670_{-8 \times 10^{-6}}^{+4 \times 10^{-6}}$ \\
\hline Orbital eccentricity, $e \ldots \ldots \ldots \ldots \ldots \ldots \ldots \ldots$ & - & $1.376(2) \times 10^{-4}$ & - \\
\hline Time of passage through periastron, $T_{0}$ (MJD) $\ldots \ldots$ & - & $56523.10321_{-6 \times 10^{-5}}^{+1 \times 10^{-5}}$ & - \\
\hline Longitude of periastron, $\omega(\operatorname{deg}) \ldots \ldots \ldots \ldots \ldots$ & - & $301.06(8)$ & - \\
\hline Time of ascending node, $T_{\text {asc }}(\mathrm{MJD}) \ldots \ldots \ldots \ldots \ldots$ & $57154.884218_{-2 \times 10^{-6}}^{+2 \times 10^{-6}}$ & - & $57243.4389465_{-1 \times 10^{-6}}^{+3 \times 10^{-7}}$ \\
\hline 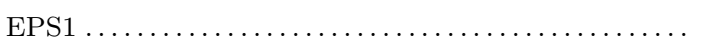 & $-2.56(87) \times 10^{-6}$ & - & $2.03(76) \times 10^{-5}$ \\
\hline 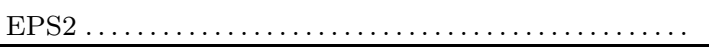 & $-0.64(74) \times 10^{-6}$ & - & $6.1(38) \times 10^{-6}$ \\
\hline \multicolumn{4}{|c|}{ Derived Quantities } \\
\hline Galactic longitude, $l(\operatorname{deg}) \ldots \ldots \ldots \ldots \ldots \ldots \ldots$ & 13.894 & 39.076 & 43.390 \\
\hline Galactic latitude, $b(\operatorname{deg}) \ldots \ldots \ldots \ldots \ldots \ldots \ldots$ & 31.831 & 10.647 & -15.765 \\
\hline 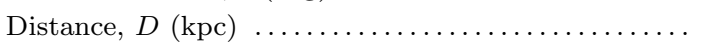 & 0.95 & 2.9 & 2.4 \\
\hline Spin period, $P(\mathrm{~s}) \ldots \ldots \ldots \ldots \ldots \ldots \ldots \ldots \ldots \ldots \ldots$ & 0.00283361387722 & 0.00406591613947 & 0.00216355679357 \\
\hline Spin period derivative, $\dot{P}\left(10^{-21} \mathrm{ss}^{-1}\right) \ldots \ldots \ldots \ldots$ & 21.33 & 5.37 & 3.29 \\
\hline $\log _{10}($ Characteristic age, yr $) \ldots \ldots \ldots \ldots \ldots \ldots \ldots$ & 9.32 & 10.08 & 10.02 \\
\hline $\log _{10}($ Surface magnetic field strength, G) & 8.40 & 8.17 & 7.93 \\
\hline $\log _{10}($ Edot, ergs/s $) \ldots \ldots \ldots \ldots \ldots \ldots$ & 34.57 & 33.50 & 34.11 \\
\hline Mass function, $f\left(M_{\odot}\right) \ldots \ldots \ldots \ldots \ldots \ldots \ldots \ldots \ldots \ldots \ldots$ & 0.0019 & 0.0070 & 0.0015 \\
\hline Minimum companion mass, $M_{c}\left(M_{\odot}\right) \ldots \ldots \ldots \ldots$ & 0.17 & 0.27 & 0.15 \\
\hline Uncalibrated flux density at $327 \mathrm{MHz}, S_{327}(\mathrm{mJy}) \ldots$ & 0.23 & 0.13 & 0.25 \\
\hline
\end{tabular}


Table 2. Parameters for the "black widow" pulsars. Notation as in Table 1. J1908+2105 spans the observational properties of the black widow and redback classes. Here and in Figure 7 it is provisionally grouped with black widows as its minimum companion mass is closer to what is typical for this type of binary system.

\begin{tabular}{|c|c|c|c|c|}
\hline \multicolumn{5}{|c|}{ Observations and data-set parameters } \\
\hline Pulsar name $\ldots \ldots \ldots \ldots \ldots \ldots \ldots \ldots \ldots \ldots \ldots \ldots \ldots$ & $\mathrm{J} 0251+2606$ & $\mathrm{~J} 1805+0615$ & $\mathrm{~J} 1908+2105$ & $\mathrm{~J} 2052+1219$ \\
\hline 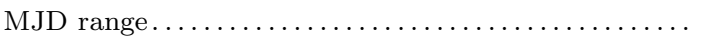 & $54682.7-58696.0$ & $54682.7-58696.0$ & $54682.7-58696.0$ & $54682.7-58696.0$ \\
\hline 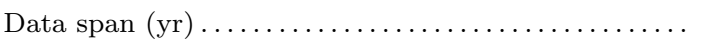 & 11.0 & 11.0 & 11.0 & 11.0 \\
\hline Reference epoch (MJD) $\ldots \ldots \ldots \ldots \ldots \ldots \ldots \ldots \ldots$ & 56644 & 57002 & 56930 & 56548 \\
\hline \multicolumn{5}{|c|}{ Measured Quantities } \\
\hline Right ascension, $\alpha$ (hh:mm:ss) $\ldots \ldots \ldots \ldots \ldots \ldots \ldots$ & $02: 51: 02.5540_{-0.0007}^{+0.0003}$ & $18: 05: 42.39969^{+3 \times 10^{-5}}$ & $19: 08: 57.2939_{-0.0002}^{+0.0004}$ & $20: 52: 47.77818^{+9 \times 10^{-5}}$ \\
\hline Declination, $\delta$ (dd:mm:ss) $\ldots \ldots \ldots \ldots \ldots \ldots \ldots \ldots$ & $+26: 06: 09.97_{-0.02}^{+0.02}$ & $+06: 15: 18.614_{-0.020}^{+0.005}$ & $+21: 05: 02.720_{-0.003}^{+0.008}$ & $+12: 19: 59.020_{-0.003}^{+0.006}$ \\
\hline Proper motion in right ascension, $\mu_{\alpha} \cos \delta\left(\operatorname{mas~yr}^{-1}\right)$ & $17(3)$ & $8.7(13)$ & - & $-4.30(32)$ \\
\hline Proper motion in declination, $\mu_{\delta}\left(\operatorname{mas} \mathrm{yr}^{-1}\right) \ldots \ldots \ldots$ & - & $12.8(29)$ & - & $-13.96(59)$ \\
\hline Dispersion measure, $\mathrm{DM}\left(\mathrm{cm}^{-3} \mathrm{pc}\right) \ldots \ldots \ldots \ldots \ldots$ & 20.2166 & 64.883 & 61.9067 & 41.9596 \\
\hline 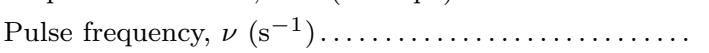 & $393.46001048595_{-5 \times 10^{-11}}^{+2 \times 10^{-11}}$ & $469.72472452336_{-1 \times 10^{-10}}^{+8 \times 10^{-11}}$ & $389.955879788271_{-9 \times 10^{-12}}^{+6 \times 10^{-11}}$ & $503.71330349547_{-3 \times 10^{-11}}^{+1 \times 10^{-11}}$ \\
\hline First derivative of pulse frequency, $\dot{\nu}\left(10^{-15} \mathrm{~Hz} \mathrm{~s}^{-1}\right)$ & $-1.1713_{-0.0002}^{+0.002}$ & $-5.0216_{-0.002}^{+0.0005}$ & $-2.1038_{-0.0006}^{+0.0006}$ & $-1.7009_{-0.0005}^{+0.0004}$ \\
\hline Binary model $\ldots \ldots \ldots \ldots \ldots \ldots \ldots \ldots \ldots \ldots \ldots$ & ELL1 & ELL1 & ELL1 & ELL1 \\
\hline Orbital period, $P_{\mathrm{b}}(\mathrm{d}) \ldots \ldots \ldots \ldots \ldots \ldots \ldots \ldots \ldots \ldots$ & $0.2024406405_{-1 \times 10^{-9}}^{+7 \times 10^{-10}}$ & $0.3368720352_{-9 \times 10^{-9}}^{+6 \times 10^{-10}}$ & $0.1463168431_{-1 \times 10^{-10}}^{+6 \times 10^{-10}}$ & $0.1146136250_{-1 \times 10^{-10}}^{+2 \times 10^{-10}}$ \\
\hline Projected semi-major axis of orbit, $x($ lt-s) $\ldots \ldots \ldots$ & $0.065678_{-5 \times 10^{-6}}^{+1 \times 10^{-5}}$ & $0.087733_{-2 \times 10^{-5}}^{+9 \times 10^{-6}}$ & $0.116895_{-2 \times 10^{-6}}^{+2 \times 10^{-5}}$ & $0.061375_{-2 \times 10^{-6}}^{+6 \times 10^{-6}}$ \\
\hline Time of ascending node, $T_{\mathrm{asc}}(\mathrm{MJD}) \ldots \ldots \ldots \ldots$ & $56647.008791_{-4 \times 10^{-6}}^{+5 \times 10^{-6}}$ & $57001.602777_{-4 \times 10^{-6}}^{+9 \times 10^{-6}}$ & $\begin{array}{r}56478.283490_{-2 \times 10^{-6}}^{+2 \times 10^{-6}} \\
-2 \times\end{array}$ & $\begin{array}{r}-2 \times 10^{-0} \\
56548.106219_{-2 \times 10^{-6}}^{+1 \times 10^{-6}}\end{array}$ \\
\hline 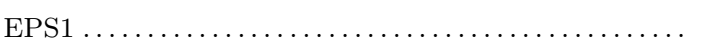 & $-3.1(76) \times 10^{-5}$ & $1.55(43) \times 10^{-4}$ & 0 & 0 \\
\hline 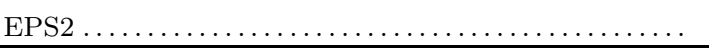 & $-1.17(65) \times 10^{-4}$ & $1.0(36) \times 10^{-5}$ & 0 & 0 \\
\hline \multicolumn{5}{|c|}{ Derived Quantities } \\
\hline Galactic longitude, $l(\mathrm{deg})$. & 153.881 & 33.351 & 53.690 & 59.139 \\
\hline Galactic latitude, $b(\operatorname{deg}) \ldots \ldots \ldots \ldots \ldots \ldots \ldots$ & -29.495 & 13.006 & 5.772 & -19.989 \\
\hline Distance, $D(\mathrm{kpc}) \quad \ldots \ldots \ldots \ldots \ldots \ldots \ldots \ldots \ldots \ldots . \ldots . \ldots . \ldots . \ldots$ & 0.96 & 3.9 & 2.6 & 3.9 \\
\hline 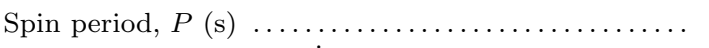 & 0.00254155434695 & 0.00212890645902 & 0.00256439267063 & 0.00198525628182 \\
\hline Spin period derivative, $\dot{P}\left(10^{-21} \mathrm{ss}^{-1}\right) \ldots \ldots \ldots \ldots$ & 7.57 & 22.76 & 13.84 & 6.70 \\
\hline $\log _{10}($ Characteristic age, yr $) \ldots \ldots \ldots \ldots \ldots \ldots$ & 9.73 & 9.17 & 9.47 & 9.67 \\
\hline $\log _{10}$ (Surface magnetic field strength, G) $\ldots \ldots \ldots$ & 8.14 & 8.35 & 8.28 & 8.07 \\
\hline $\log _{10}($ Edot, ergs $/ s) \ldots \ldots \ldots \ldots \ldots \ldots \ldots \ldots \ldots \ldots \ldots$ & 34.26 & 34.97 & 34.51 & 34.53 \\
\hline 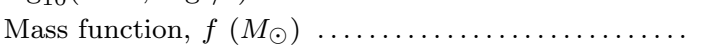 & $7.4 \times 10^{-6}$ & $6.4 \times 10^{-6}$ & $8.0 \times 10^{-5}$ & $1.9 \times 10^{-5}$ \\
\hline Minimum companion mass, $M_{c}\left(M_{\odot}\right) \ldots \ldots \ldots \ldots$ & 0.025 & 0.023 & 0.055 & 0.034 \\
\hline Uncalibrated flux density at $327 \mathrm{MHz}, S_{327}(\mathrm{mJy}) \ldots$ & 0.17 & 0.43 & 0.19 & 0.52 \\
\hline
\end{tabular}


Table 3. Parameters for the eclipsing "redback" pulsar J1048+2339. Notation as in Table 1.

\begin{tabular}{|c|c|}
\hline \multicolumn{2}{|l|}{ Observation and data-set parameters } \\
\hline Pulsar name. & $\mathrm{J} 1048+2339$ \\
\hline MJD range...$\ldots \ldots \ldots \ldots \ldots \ldots \ldots \ldots \ldots \ldots \ldots \ldots$ & $56508.8-57548.9$ \\
\hline 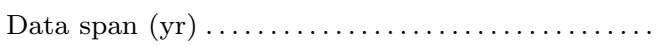 & 2.85 \\
\hline Epoch of frequency determination (MJD) ........ & 57285 \\
\hline Epoch of position determination (MJD) ......... & 56700 \\
\hline Epoch of dispersion measure determination (MJD) & 56700 \\
\hline \multicolumn{2}{|l|}{ Measured Quantities } \\
\hline Right ascension, $\alpha$ (hh:mm:ss) $\ldots \ldots \ldots \ldots \ldots \ldots$ & 10:48:43.418354(76) \\
\hline Declination, $\delta$ (dd:mm:ss) $\ldots \ldots \ldots \ldots \ldots \ldots$ & $+23: 39: 53.4043(20)$ \\
\hline Proper motion in $\alpha, \mu_{\alpha} \cos \delta\left(\operatorname{mas~yr}^{-1}\right) \ldots \ldots \ldots$ & $-18.7^{a}$ \\
\hline Proper motion in $\delta, \mu_{\delta}\left(\operatorname{mas~yr}^{-1}\right) \ldots \ldots$ & $-9.4^{a}$ \\
\hline Dispersion measure, DM $\left(\mathrm{cm}^{-3} \mathrm{pc}\right) \ldots$ & 16.6543 \\
\hline Pulse frequency, $\nu\left(\mathrm{s}^{-1}\right) \ldots \ldots \ldots \ldots$ & $214.35478534113(2)$ \\
\hline First derivative of $\nu, \dot{\nu}\left(10^{-15} \mathrm{~Hz} \mathrm{~s}^{-2}\right) \ldots \ldots \ldots \ldots$ & $-1.382(2)$ \\
\hline Binary model................................ & BTX \\
\hline Orbital period $(\mathrm{d})$ & $0.2505191 b$ \\
\hline Projected semi-major axis of orbit, $x$ (lt-s). & $0.836120(3)$ \\
\hline Epoch of periastron, $T_{0}$ (MJD) $\ldots \ldots \ldots \ldots$ & $56637.598174(2)$ \\
\hline Longitude of periastron, $\omega_{0}(\mathrm{deg}) \ldots \ldots \ldots \ldots \ldots$ & 0 \\
\hline Orbital eccentricity, $e \ldots \ldots \ldots \ldots \ldots \ldots \ldots \ldots$ & 0 \\
\hline Orbital frequency, $F B 0(\mathrm{~Hz}) \ldots \ldots \ldots \ldots \ldots \ldots$ & $4.6200355(5) \times 10^{-5}$ \\
\hline 1st orbital frequency derivative, $F B 1\left(\mathrm{~Hz} \mathrm{~s}^{-1}\right) \ldots$ & $-4.18(55) \times 10^{-18}$ \\
\hline 2nd orbital frequency derivative, $F B 2\left(\mathrm{~Hz} \mathrm{~s}^{-2}\right) \ldots$ & $1.4(12) \times 10^{-25}$ \\
\hline 3rd orbital frequency derivative, $F B 3\left(\mathrm{~Hz} \mathrm{~s}^{-3}\right) \ldots$ & $-1.50(61) \times 10^{-31}$ \\
\hline 4th orbital frequency derivative, $F B 4\left(\mathrm{~Hz} \mathrm{~s}^{-4}\right) \ldots$ & $8.0(13) \times 10^{-38}$ \\
\hline 5 th orbital frequency derivative, $F B 5\left(\mathrm{~Hz} \mathrm{~s}^{-5}\right) \ldots$ & $-1.65(18) \times 10^{-44}$ \\
\hline 6 th orbital frequency derivative, $F B 6\left(\mathrm{~Hz} \mathrm{~s}^{-6}\right) \ldots$ & $1.83(17) \times 10^{-51}$ \\
\hline 7 th orbital frequency derivative, $F B 7\left(\mathrm{~Hz} \mathrm{~s}^{-7}\right) \ldots$ & $-1.119(91) \times 10^{-58}$ \\
\hline 8th orbital frequency derivative, $F B 8\left(\mathrm{~Hz} \mathrm{~s}^{-8}\right) \ldots$ & $3.00(23) \times 10^{-66}$ \\
\hline \multicolumn{2}{|l|}{$\begin{array}{l}\text { Derived Quantities } \\
\end{array}$} \\
\hline Galactic longitude, $l(\operatorname{deg}) \ldots \ldots \ldots \ldots \ldots \ldots$ & 32.709 \\
\hline Galactic latitude, $b(\operatorname{deg}) \ldots \ldots \ldots \ldots \ldots \ldots$ & 62.139 \\
\hline Distance, $D(\mathrm{kpc}) \ldots \ldots \ldots \ldots \ldots$ & 1.7 \\
\hline Spin period, $P(\mathrm{~s}) \ldots \ldots \ldots \ldots \ldots \ldots$ & 0.00466516293727 \\
\hline Spin period derivative, $\dot{P}\left(10^{-21} \mathrm{~s} \mathrm{~s}^{-1}\right) \ldots$ & 30.07 \\
\hline $\log _{10}($ Characteristic age, yr $) \ldots \ldots \ldots \ldots \ldots$ & 9.39 \\
\hline $\log _{10}($ Surface magnetic field strength, G) $\ldots \ldots$ & 8.58 \\
\hline $\log _{10}($ Edot, ergs $/ \mathrm{s}) \ldots \ldots \ldots \ldots \ldots \ldots \ldots \ldots \ldots \ldots$ & 34.07 \\
\hline Mass function, $f\left(M_{\odot}\right) \ldots \ldots \ldots \ldots \ldots \ldots \ldots$ & 0.01 \\
\hline Minimum companion mass, $M_{c}\left(M_{\odot}\right) \ldots \ldots \ldots$ & 0.31 \\
\hline Uncalibrated flux density at $327 \mathrm{MHz}, S_{327}$ (mJy) & 0.42 \\
\hline
\end{tabular}

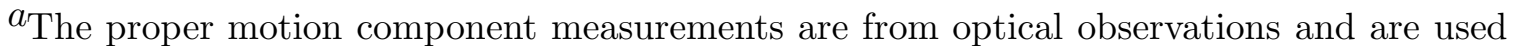
as fixed parameters in the timing solution (see Deneva et al. 2016, Section 6.2 for detailed discussion).

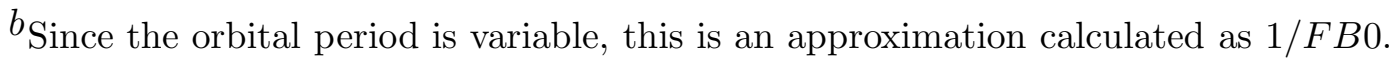




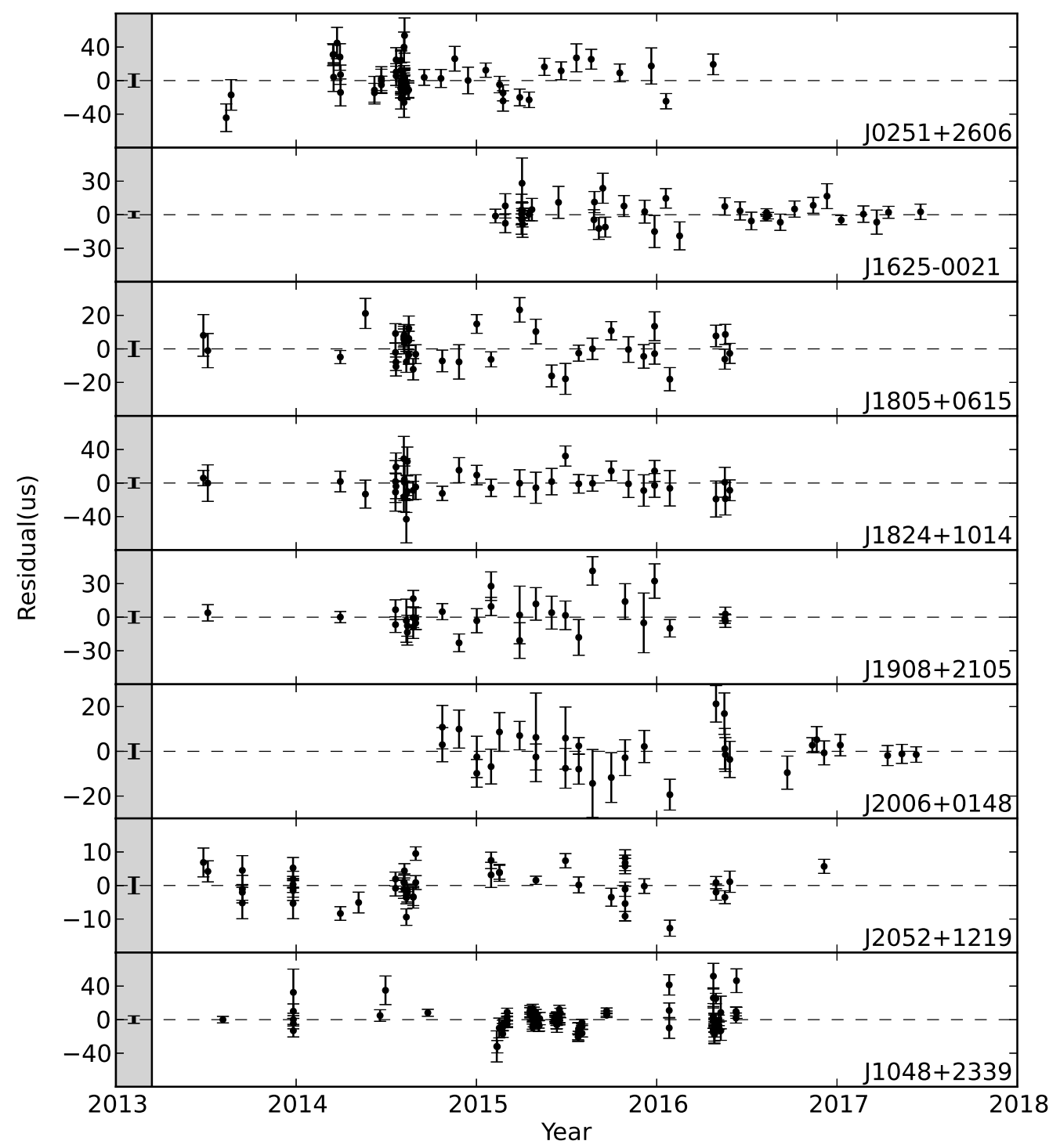

Figure 1. Radio timing residuals vs. observing epoch. All observations are at $327 \mathrm{MHz}$. The error bar within the shaded area on the left of each panel shows the size of the weighted root-mean-square residual.

\subsection{PSR J1805+0615}

This black widow MSP was discovered in the Fermi-LAT source 3FGL J1805.9+0614 (4FGL J1805.6+0615). The radio timing residuals vs. observing epoch are shown in Figure 1. The Fermi timing solution is shown in Table 2. The pulsar has an orbital period of 8.08 hours and a minimum companion mass of $0.023 \mathrm{M}_{\odot}$. Figure 3 (bottom left) shows the photon phaseogram and folded gamma- 


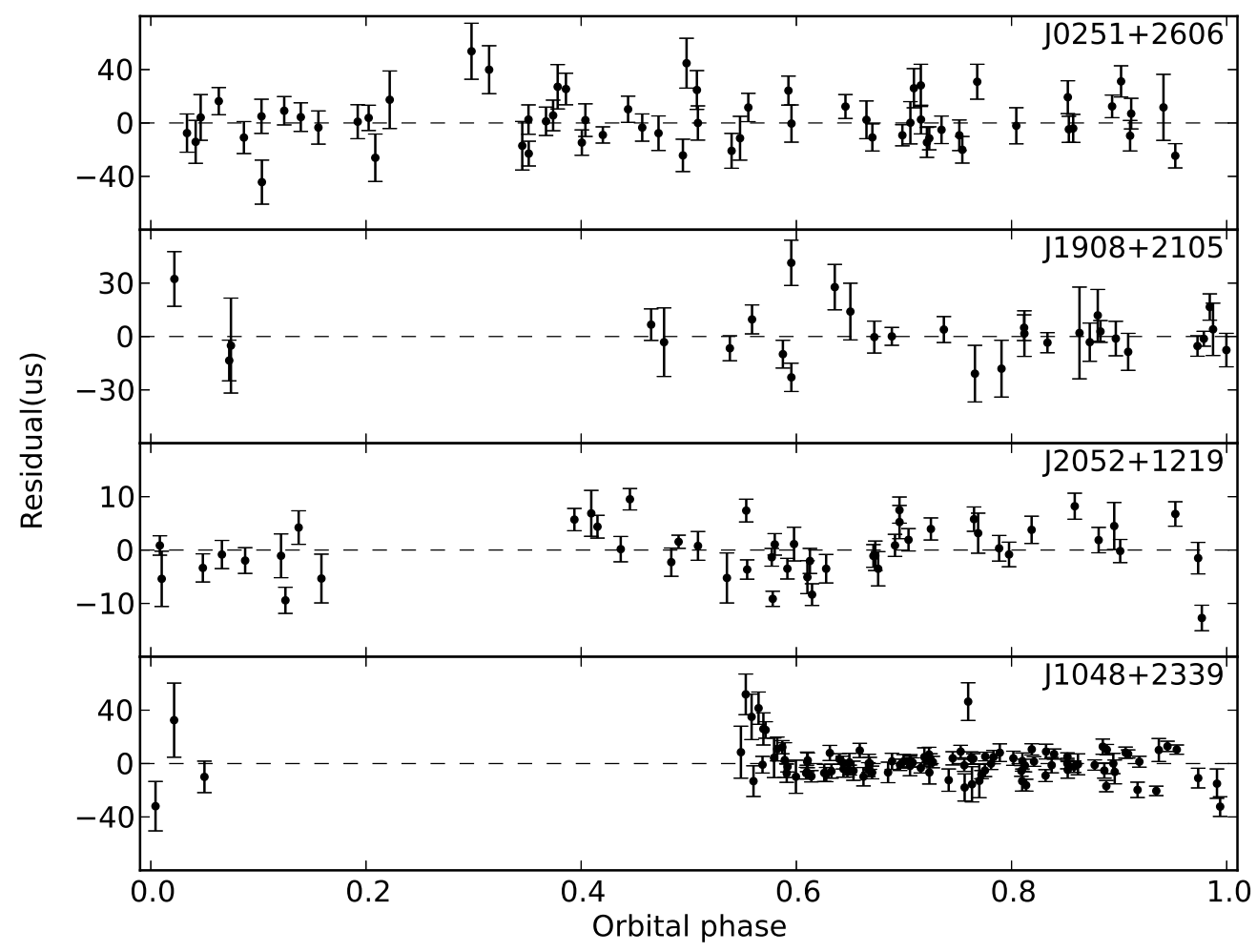

Figure 2. Radio timing residuals vs. orbital phase for the four eclipsing pulsars in this work. All observations are at $327 \mathrm{MHz}$.

ray profile phase-aligned with the $327 \mathrm{MHz}$ radio profile. Gamma-ray pulsations were detected with a H-test of $183.29 \sim 12 \sigma$.

\subsection{PSR J1824+1014}

This MSP was discovered in the Fermi-LAT source 3FGL J1824.0+1017 (4FGL J1824.1+1013). The radio timing residuals vs. observing epoch are shown in Figure 1. The Fermi timing solution is shown in Table 1. The pulsar has an orbital period of 82.5 days, the longest presented in this work. While long- $P_{\mathrm{b}}$ binaries appear to be rare among those found in Fermi-LAT unidentified sources, the opposite is true for MSPs found in untargeted pulsar surveys; Section 5 discusses this discrepancy in more detail. This pulsar's minimum companion mass is $0.27 \mathrm{M}_{\odot}$, indicating a white dwarf companion. Figure 3 (bottom right) shows the photon phaseogram and folded gamma-ray profile phase-aligned with the $327 \mathrm{MHz}$ radio profile. Gamma-ray pulsations are weak and were detected with a H-test of $52.55(6.15 \sigma)$.

\subsection{PSR J1908+2105}

This eclipsing MSP was discovered in a source from a preliminary version of the 3FGL Fermi-LAT catalog (P7R4J1909+2102). The source was not included in the 

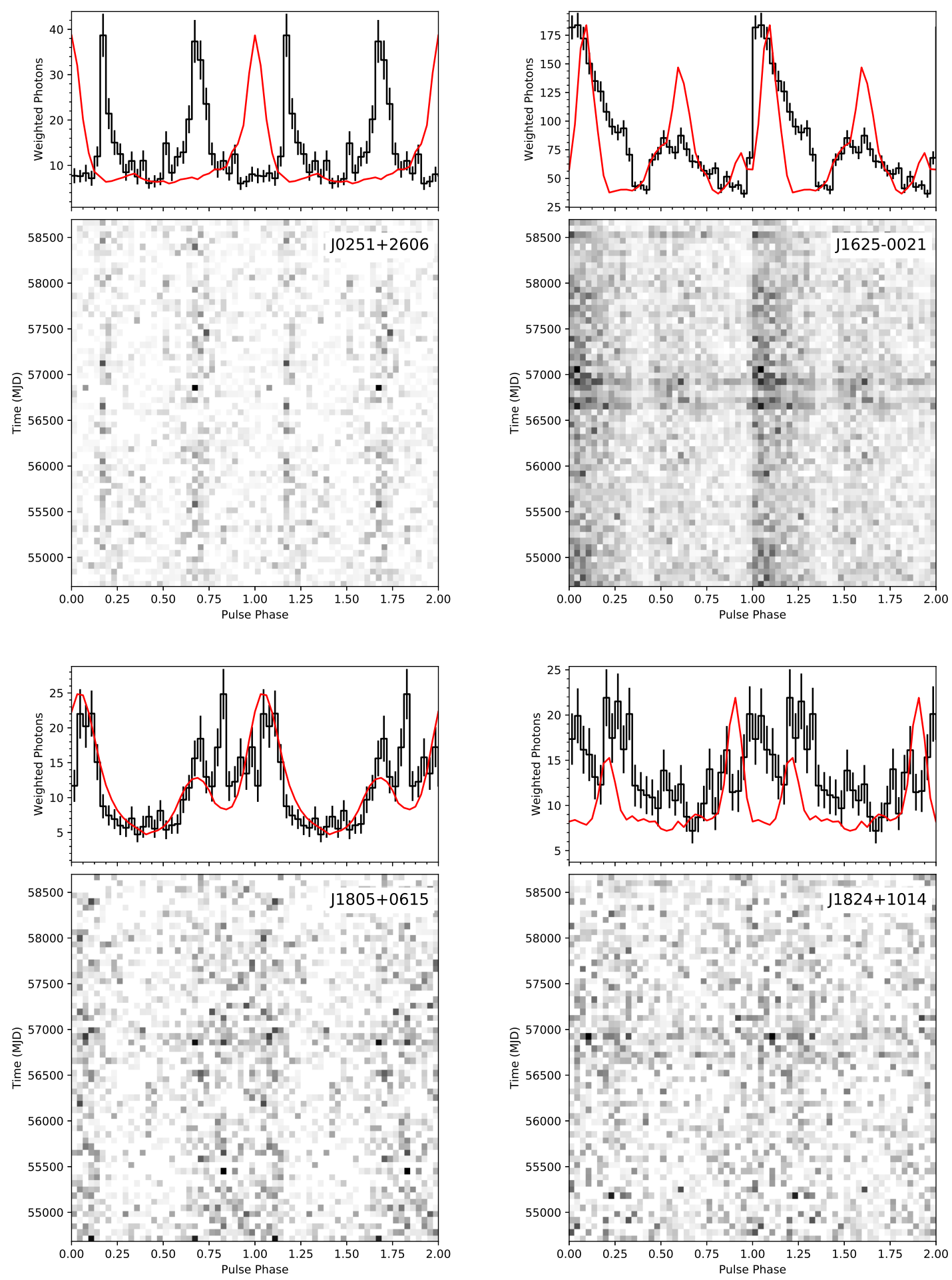

Figure 3. Weighted Fermi-LAT photon phaseograms (lower panels) and average pulse profiles (top panels) for PSRs J0251+2606 (upper left), J1625-0021 (upper right), J1805+0615 (lower left) and J1824+1014 (lower right). The phase-aligned $327 \mathrm{MHz}$ radio pulse profile is shown in red in the top panels. A temporary change in the Fermi all-sky survey strategy at MJD 56500-57000 caused a change in average exposure depending on source position. This is visible as darker horizontal bands in some of the greyscale plots. 

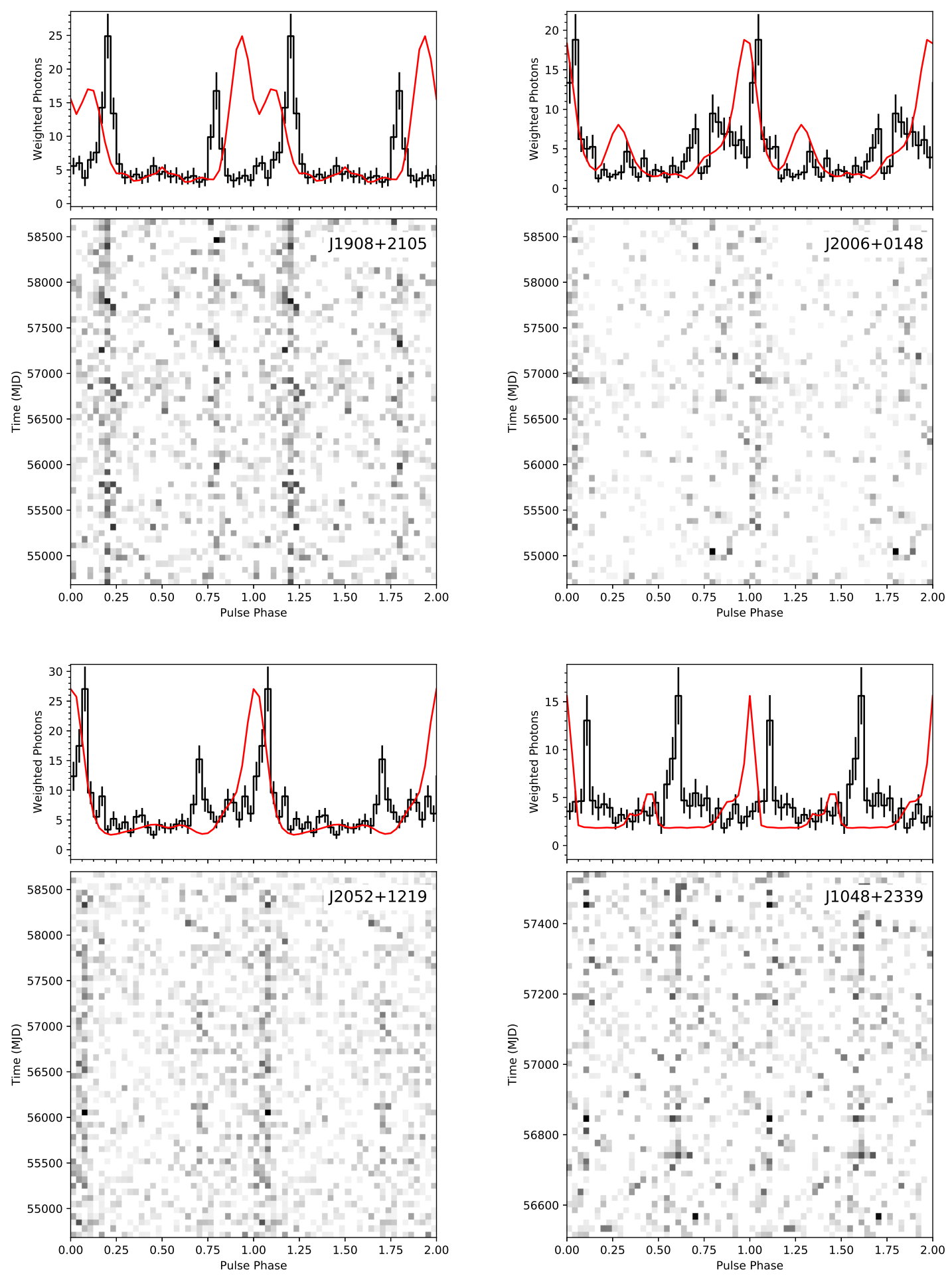

Figure 4. Same as Figure 3, for PSRs J1908+2105 (top left), J2006+0148 (top right), J2052+1219 (lower left) and J1048+2339 (lower right). 

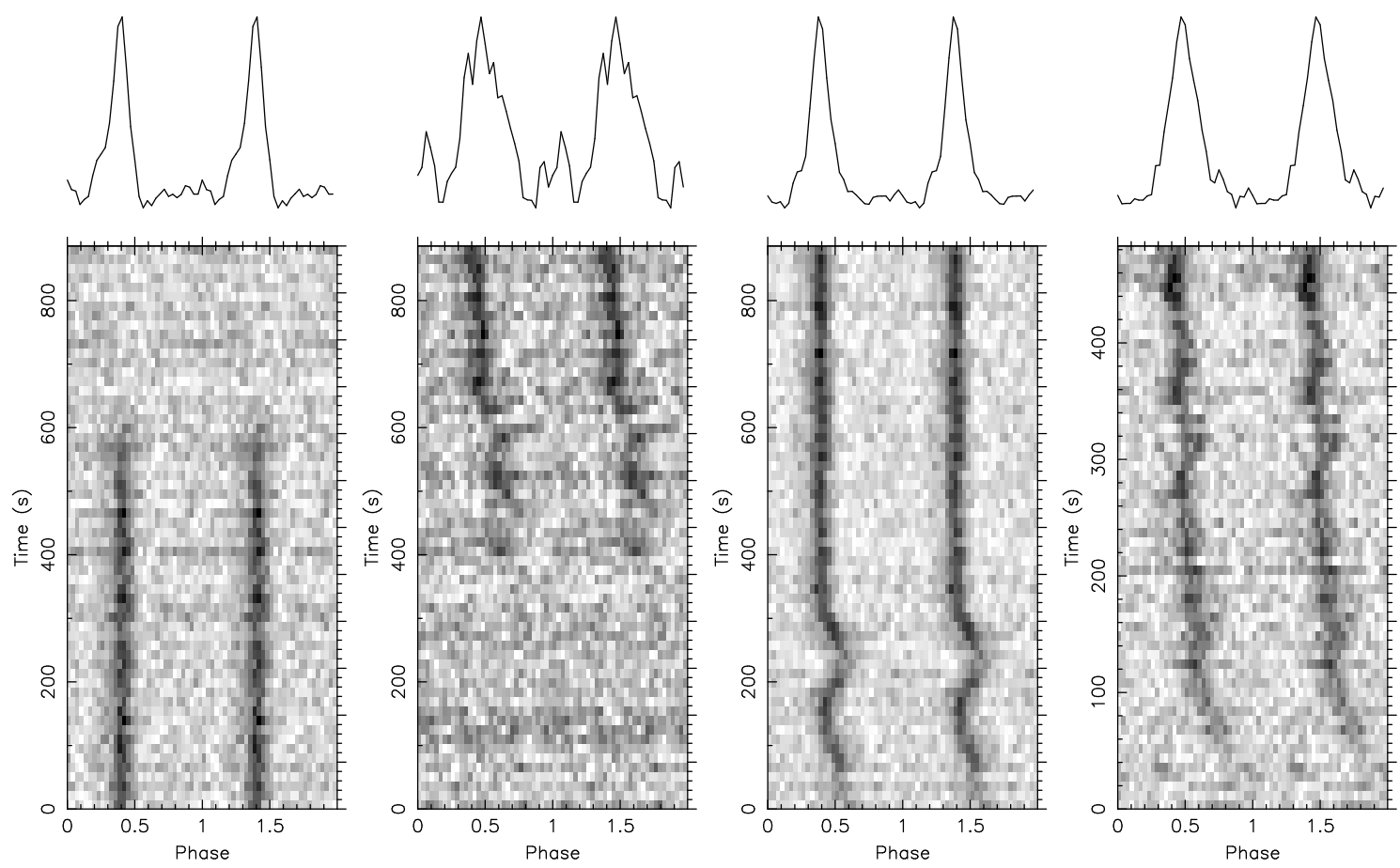

Figure 5. Four observations of J2052+1218 made with Arecibo at $327 \mathrm{MHz}$. The left two panels show ingress and egress on the same orbit (MJD 56650). The respective orbital phase ranges are $0.14-0.23$ and $0.32-0.41$. The right two panels show egress from two separate observations (MJD 56859 and 57109, orbital phases $0.29-0.38$ and $0.36-0.41$, respectively). TOAs from ingress and egress regions were not included in the timing solution due to the extra pulse delays.

final version of 3FGL, but is included in the newer 4FGL Fermi-LAT source catalog as 4FGL J1908.9+2103. The radio timing residuals vs. observing epoch and orbital phase are shown in Figures 1 and 2. The Fermi timing solution is shown in Table 3. The pulsar has an orbital period of 3.51 hours and a minimum companion mass of $0.055 \mathrm{M}_{\odot}$. While the short orbital period and relatively small companion mass are typical for black widows, its extended eclipses are typical for redbacks (see Section 5 for a more detailed discussion). Figure 4 (top left) shows the photon phaseogram and folded gamma-ray profile phase-aligned with the $327 \mathrm{MHz}$ radio profile. Gamma-ray pulsations were detected with a H-test of 374.66, corresponding to a significance of $\sim 18 \sigma$.

\subsection{PSR J2006+0148}

This MSP was discovered in the Fermi-LAT source 3FGL J2006.6+0150 (4FGL J2006.4+0147). The radio timing residuals vs. observing epoch are shown in Figure 1. The Fermi timing solution is shown in Table 1. The pulsar has an orbital period of 15.63 hours and a minimum companion mass of $0.15 \mathrm{M}_{\odot}$, indicating a white dwarf companion. Figure 4 (top right) shows the photon phaseogram and folded gamma-ray profile phase-aligned with the $327 \mathrm{MHz}$ radio profile. Gamma-ray pulsations were detected with a H-test of $216.40(13.20 \sigma)$. 


\subsection{PSR J2052+1219}

The pulsar was discovered in the Fermi-LAT source 3FGL J2052.7+1217 (4FGL J2052.7+1218). The radio timing residuals vs. epoch and orbital phase are shown in Figures 1 and 2, and the Fermi timing solution is shown in Table 2. This black widow binary has an orbital period of $2.75 \mathrm{~h}$ and a minimum companion mass of $0.034 \mathrm{M}_{\odot}$. The pulsar exhibits radio eclipses and variable delays in the radio pulse arrival time near ingress and egress (Figure 5). The relatively abrupt ingress indicates a sharp leading edge of the companion. In contrast, during the first few minutes after every observed egress we see pulse delays of up to 0.25 in rotational phase. This indicates the pulses experience an extra dispersive delay while passing through a cloud of ionized gas that trails the companion. After optimizing the timing solution over the full span of the Fermi mission, gamma-ray pulsations were detected with an Htest significance of 254.67, corresponding to $14.42 \sigma$. The phaseogram is presented in Figure 4 (bottom left).

Zharikov et al. (2019) detect an optical counterpart to PSR J2052+1218 and estimate the companion to have a radius of $0.12-0.15 R_{\odot}$, nearly filling its Roche lobe. An independent estimate of the distance to this system from the optical data is $3.94(12) \mathrm{kpc}$, which is consistent with the less precise distance estimate of $3.9 \mathrm{kpc}$ from the pulsar's DM and the YMW16 model of ionized gas in the Galaxy (Yao et al. 2017). (Dispersion-based distances have typical uncertainties of up to $40 \%$.)

\subsection{PSR J1048+2339}

An initial timing solution for this pulsar based on two years of Arecibo and GBT data was presented in Deneva et al. (2016). The precise timing position allowed the identification of a variable optical counterpart, an M4 main sequence star asymmetrically heated by the pulsar wind. The timing solution also uncovered rapid quasiperiodic oscillations in the orbital period likely due to changes in the gravitational quadrupole moment of the companion. Because of these oscillations it was not feasible to extrapolate the pulsar ephemeris outside the range of radio observations. Analyzing Fermi-LAT data within its 2-year range did not yield a detection of gamma-ray pulsations at the time. In Table 3 we present an updated timing solution based on 2.9 years of radio data that includes three additional orbital period derivatives. Figures 1 and 2 show the radio timing residuals vs. observing epoch and orbital phase. A detection of gamma-ray pulsations in Fermi-LAT data from the same time range is shown in Figure 4. The weighted H-test significance of the detection is 84.95, which corresponds to $\sim 8 \sigma$; the phaseogram is presented in Figure 4 (bottom right).

\section{DISCUSSION}

The present work continues the trend of pulsar searches in Fermi-LAT unidendified sources discovering a disproportionate number of redback and black widow systems with orbital periods of a few hours. Many of these systems exhibit radio eclipses, as is the case for four of our eight MSPs (Figure 2). 


\subsection{Targeted vs. Untargeted MSP Searches and Discoveries}

A total of 18 black widows and 7 redbacks (not including unpublished ones) have been found since 2008 in radio searches of Fermi-LAT unidentified sources. In contrast, there are 15 field black widows and 4 field redbacks in ATNF whose discovery was unrelated to Fermi and took place over the five decades since the first binary pulsar was discovered. This raises the question of whether MSP gamma-ray emission is intrinsically correlated with a short orbital period or this apparent dependence is due to selection effects. Known gamma-loud MSPs have higher $\dot{E}$ on average than MSPs detected only in radio (Abdo et al. 2013). We assembled two samples of binary MSPs whose $\dot{E}$ is known $^{5}$ and have a rotation period $P<10 \mathrm{~ms}$ : those found in FermiLAT unidentified sources, and all other field MSPs in ATNF meeting these criteria. Figure 6 shows histograms of the two field MSP samples vs. orbital period $\left(P_{\mathrm{b}}\right)$ and $\dot{E}$. Both sets of panels show visibly different distributions. We perform AndersonDarling tests on the $P_{\mathrm{b}}$ and $\dot{E}$ distributions of the two samples, confirming that they are significantly different (same-distribution null hypothesis probabilities $<0.001$, i.e. lower than the floor of probability values reported by scipy . stats . anderson_ksamp). Kolmogorov-Smirnov tests on the $P_{\mathrm{b}}$ and $\dot{E}$ distributions of the two samples using scipy.stats.ks_2samp concur, yielding same-distribution null hypothesis probabilities of $2 \times 10^{-5}$ and $1 \times 10^{-6}$, respectively.

MSPs are typically found in targeted or untargeted observations which are searched for pulsed signals assuming a range of constant line-of-sight accelerations of the pulsed source with respect to the telescope. The assumption of constant acceleration begins to break down for $P_{\mathrm{b}} \lesssim 10 \times T_{\text {obs }}$. We therefore overlay vertical lines on the two panels of Figure 6 corresponding to $10 \times T_{\text {obs }}$ of standard search observations that have yielded MSP discoveries in both of our samples. Targeted radio observations of Fermi-LAT unidentified sources (top panels) tend to be $15-60$ minutes, while untargeted radio pulsar survey observations (bottom panels) tend to be $1-5$ minutes. We see that in the top left panel, MSP discoveries drop off drastically for $P_{\mathrm{b}}$ smaller than the shortest $10 \times T_{\text {obs }}$ of targeted search observations. This indicates that new discoveries are potentially acceleration-limited. It also suggests that such observations should be searched not only as one continuous $T_{\text {obs }}$ but also separately in chunks of a shorter duration, down to 5 minutes, which would provide some continuity with untargeted surveys in acceleration-limited sensitivity. Another approach is to use jerk searches, which do not assume a constant line-of-sight acceleration but add its first derivative as an extra search dimension (Tabassum et al. 2020).

In the bottom left panel in contrast, there is a gap showing no MSP discoveries over a range of $P_{\mathrm{b}}>10 \times T_{\mathrm{obs}}$. This indicates that untargeted surveys are likely flux-limited, in several different ways. First, the minimum detectable flux density,

\footnotetext{
${ }^{5}$ Values of $\dot{E}$ used throughout this work are not corrected for the Shklovskii effect. This choice avoids heterogeneity in our comparison samples as many MSPs found in Fermi-LAT unidentified sources do not have the proper motion measurements needed for the correction, including four of the eight in this work.
} 

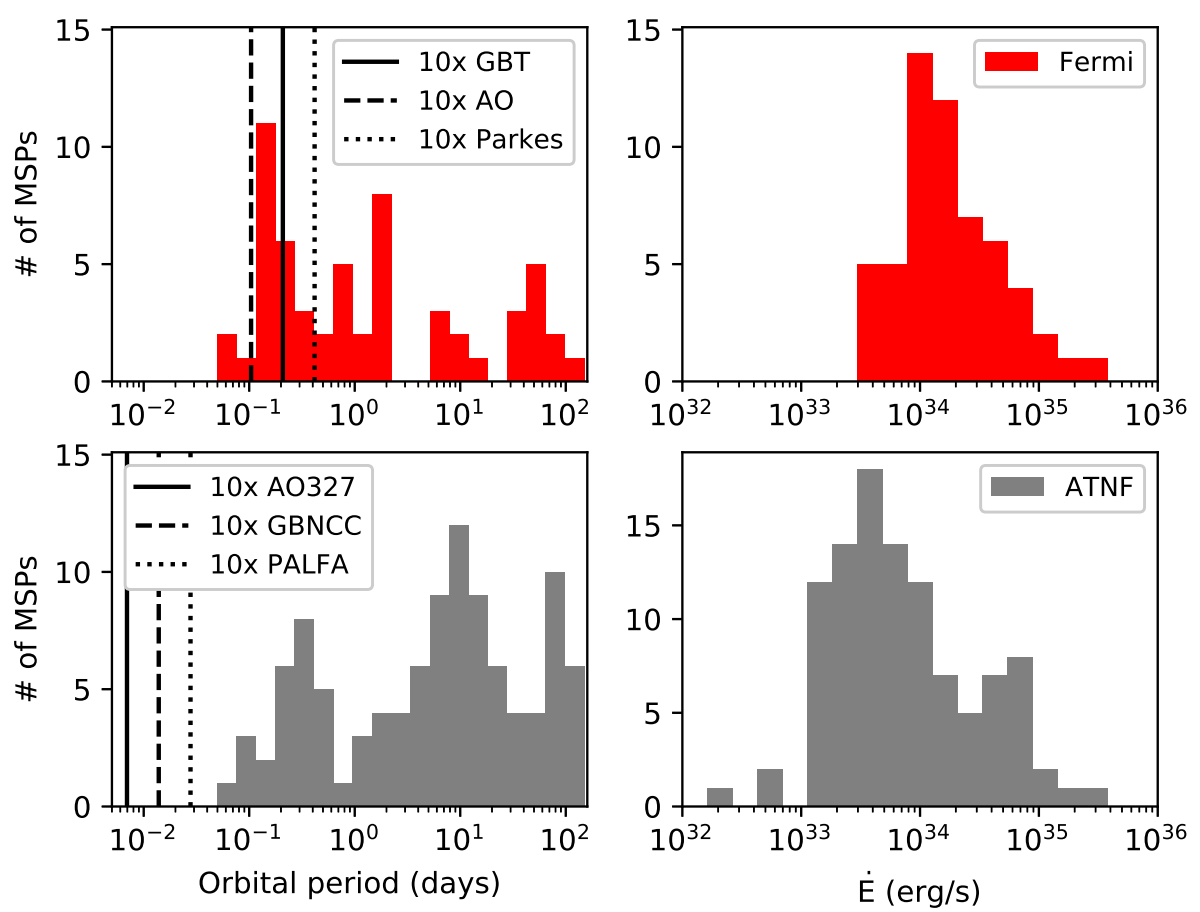

Figure 6. Top: Histograms of MSPs found in searches of Fermi-LAT unidentified sources vs orbital period (left) and $\dot{E}$ (right). In the top left panel vertical lines show $10 \times T_{\text {obs }}$ for searches of such sources at three observatories. Bottom: The same histograms for field MSPs from ATNF, excluding those found in Fermi-LAT unidentified sources. In the bottom left panel vertical lines show $10 \times T_{\text {obs }}$ for several untargeted radio pulsar surveys. Searches for binary pulsars assume constant line-of-sight acceleration; this assumption begins to break down for $P_{\mathrm{b}}<10 \times T_{\mathrm{obs}}$.

$S_{\min } \propto 1 / \sqrt{T_{\mathrm{obs}}}$, which puts shorter survey observations at a disadvantage compared to longer targeted ones. In untargeted surveys the center of the nearest radio beam would be offset from the (unknown) pulsar position by up to the half-power beam radius, which degrades $S_{\text {min }}$ by up to a factor of 2 . Unidentified Fermi-LAT sources are selected for targeted pulsar searches so that their error ellipses fit within the radio beam, and the beam center is placed at the error ellipse center, making any potential offset from the actual pulsar position likely much smaller than the beam radius.

The spike of MSPs with short $P_{\mathrm{b}}$ in the top left panel of Figure 6 is matched by a decline in the bottom left panel. Untargeted surveys cover a position on the sky only once, while targeted searches of Fermi-LAT unidentified sources observe every source of interest several times. This means that the latter are more likely to detect heavily eclipsing, short- $P_{\mathrm{b}}$ MSPs like J1048+2339 and J1908+2105 from the present work. On the other hand, a spike of MSPs with $P_{\mathrm{b}}$ on the order of a few days in the bottom left panel is matched by a scarcity of such discoveries in Fermi-LAT sources in the top left panel. This can be explained by the fact that there is a historic bias in favor of long- $P_{\mathrm{b}}$ pulsars whose detectability is not significantly affected by line-of-sight 
acceleration. Many of these pulsars were easily detectable without and discovered before the advent of acceleration searches. A number of untargeted surveys from the past few decades with $T_{\text {obs }}$ ranging from a few minutes to an hour or longer have had a long head start in searching this part of the parameter space before targeted searches of Fermi-LAT sources began.

We conclude that selection effects are prominent enough that they cannot be ruled out as explanation for the different $P_{\mathrm{b}}$ distributions of MSPs found in Fermi-LAT unidentified sources compared to MSPs found in untargeted all-sky surveys. Despite the different selection biases affecting our two MSP samples, there is a notable lack

of discoveries with $P_{\mathrm{b}} \lesssim 1$ hour in both, even though some modern surveys using acceleration and jerk searches are in principle sensitive to signals in that part of the parameter space. In such cases targeted and untargeted radio pulsar searches alike may be hindered by an extreme manifestation of the effects making some redbacks and black widows difficult to detect: heavy eclipses, prolonged and uneven dispersive pulse delays like those shown in Figure 5, and intense scattering by ionized gas in the system. Dispersive and scattering effects would make even non-eclipsing pulsars with very short orbital periods difficult to detect. Since gamma-ray emission is unaffected by dispersion and scattering, binary MSPs with $P_{\mathrm{b}} \lesssim 1$ hour may stand a better chance of detection when direct searches for gamma-ray pulsations from MSPs become computationally feasible. This is exemplified by the two MSPs with the shortest orbital periods known, both of them black widow binaries: PSR J1311-3430 $\left(P_{b}=\right.$ $93 \mathrm{~min}$; Pletsch et al. 2012a), which is very rarely detected at radio wavelengths, and PSR J0653-0158 ( $P_{b}=75 \mathrm{~min}$; Nieder at al. 2020), which has not been detected at radio wavelengths.

\subsection{MSP Binary Types}

Tauris \& Savonije (1999) derived a relation between the orbital period $P_{\mathrm{b}}$ and companion mass $M_{c}$ for three populations of NS-WD binary systems depending on the metallicity of the WD progenitor. Assuming a pulsar mass of $1.4 \mathrm{M}_{\odot}$ Figure 7 shows where our MSPs fall with respect to these relations based on their minimum companion masses (corresponding to an orbital inclination of $90^{\circ}$ ) as well as the companion mass corresponding to the median orbital inclination of $60^{\circ}$. Of the three NS-WD systems from Table $1 \mathrm{~J} 1824+1014$ and J2006+0148 conform well to the relation, while J1625-0021 does not. This indicates that the orbital plane of J1625-0021 may be less inclined than the other two systems: an inclination of $\sim 43^{\circ}-45^{\circ}$ corresponds to a $M_{c}=0.24-0.25 \mathrm{M}_{\odot}$, which would be consistent with the theoretical relation. Another possibility is that the neutron star in the J1625-0021 system is more massive than the assumed pulsar mass of $1.4 \mathrm{M}_{\odot}$. For the median orbital inclination of $60^{\circ}$, a pulsar mass of $1.9-2.1 \mathrm{M}_{\odot}$ would also result in this system conforming to the relation. 
Redbacks fall in the lower right corner and black widows in the lower left corner of Figure 7. Cromartie et al. (2016) classified J1048+2339 and J1908+2105 as redbacks based on the fact that both pulsars eclipse for nearly half of their orbital periods (Figure 2). Heavy eclipses are typical for redback systems due to their similarly short orbital periods but larger companions compared to black widow systems. In both types of binaries the intense pulsar particle wind gives rise to a plume of ionized gas around the companion that further contributes to the eclipse duration.

Figure 7 shows $M_{c}$ for the redback J1048+2339 with the same assumed pulsar mass and two inclinations as for the other MSPs in this work. It also shows a spectroscopic result from Strader et al. (2019), who argue that the pulsar mass in this system is $\geq 1.96 \mathrm{M}_{\odot}$, and $M_{c} \geq 0.38 \mathrm{M}_{\odot}$. The inclination corresponding to these parameters is $\geq 83_{-10}^{+7}$ degrees. Therefore either the orbit must be close to edge-on, or the pulsar mass must be signifcantly higher than $2 \mathrm{M}_{\odot}$.

Even though J1908+2105 was previously classified as a redback, its short orbital period and relatively small minimum $M_{c}$ place it in the lower left corner in Figure 7 together with the black widows from Table 2. However, the fact that J1908+2105 eclipses for $\sim 40 \%$ of its orbit is unusual for a black widow. When black widows eclipse, they do so for no more than $\sim 10-20 \%$ of their orbital periods (e.g. Polzin et al. 2018, Polzin et al. 2019, Crowter et al. 2020). This points either to an atypical companion size in the J1908+2105 system and/or an unusually dense and extended ionized gas halo around the companion. Alternatively, J1908+2105 could represent a middle-ground case between the two observational classes of redbacks and black widows. Chen et al. (2013) deduce from stellar evolution simulations that redbacks do not evolve into black widows, but the bimodal distribution of properties between these two binary pulsar types is due to how efficiently the pulsar wind irradiates the companion, which in turn depends on beaming geometry.

Another useful comparison is to see where our MSPs fall with respect to the theoretical relation between eccentricity and orbital period in NS-WD systems derived by Phinney (1992) and expanded by Phinney \& Kulkarni (1994). In Figure 8 the median values and $95 \%$ uncertainty of this relation are shown for a range of typical orbital periods. For a comparable companion mass, binaries with wider orbits would take longer to tidally circularize. However, Phinney (1992) and Phinney \& Kulkarni (1994) show that if tidal forces were the only or the dominant factor determining the final eccentricity in the system, most NS-WD binaries should have eccentricities indistinguishable from zero. The tidal circularization time scale is shorter than the giant evolution stage of the companion by several orders of magnitude; during this stage the companion fills its Roche lobe and mass transfer to the NS is ongoing. Instead, the effect of tides on orbital evolution is partly counteracted by eddies and density fluctuations within the convective layer of the giant, such that after mass transfer stops and the companion reaches its WD stage, the eccentricity remains "frozen" at a small but measurably non-zero value. 


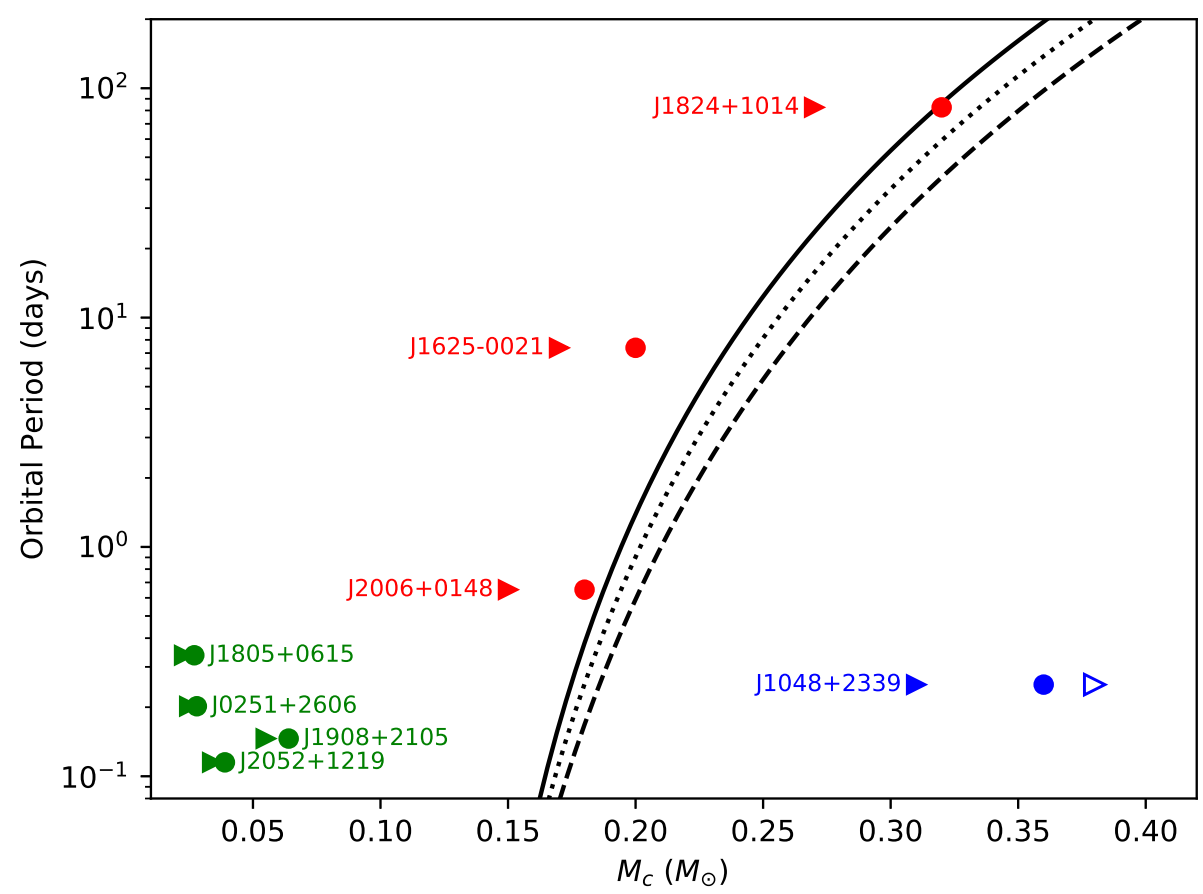

Figure 7. Curves show the $P_{\mathrm{b}}-M_{c}$ relation for the three populations of NS-WD binaries based on the metallicity of the WD progenitor considered by Tauris \& Savonije (1999). Red triangles show the minimum companion masses for the three NS-WD systems in this work. Red circles show the companion mass for the median orbital inclination of $60^{\circ}$. Green and blue symbols correspond to the black widow and redback systems in this work, respectively. Filled symbols are based on pulsar timing in the present work and assume a pulsar mass of $1.4 \mathrm{M}_{\odot}$. The sole open triangle denotes a spectroscopic result from Strader et al. (2019) for the companion mass of $\mathrm{J} 1048+2339$, corresponding to a pulsar mass of $1.96 \mathrm{M}_{\odot}$ and inclination of $83^{\circ}$.

Five of our eight MSPs have non-zero eccentricities in their best-fit timing solutions. Figure 8 shows that two of the NS-WD systems in Table 1 (J1625-0021 and $\mathrm{J} 1824+1014$ ) conform well to the relation between eccentricity and orbital period, while the third $(\mathrm{J} 2006+0148)$ does not. The relation was derived assuming stable mass transfer from a giant star filling its Roche lobe. Such systems are expected to have $P_{\mathrm{b}}>1$ day and $0.16 \mathrm{M}_{\odot} \lesssim M_{c} \lesssim 0.45 \mathrm{M}_{\odot}$ in their NS-WD stage. J1625-0021 and $\mathrm{J} 1824+1014$ have $P_{\mathrm{b}}=7$ and 83 days, respectively, while $\mathrm{J} 2006+0148$ has $P_{\mathrm{b}}=15.6$ hours. Of the three MSPs J1824+1014 has the highest minimum companion mass, $M_{c}>0.27 \mathrm{M}_{\odot}$, while $\mathrm{J} 1625-0021$ and $\mathrm{J} 2006+0148$ have $M_{c}>0.17$ and $0.15 \mathrm{M}_{\odot}$, respectively. We conclude that the properties of J2006+0148 indicate a relatively short or intermittent mass transfer from a companion whose giant stage did not last long enough for the orbit to become very circularized.

\section{CONCLUSIONS}




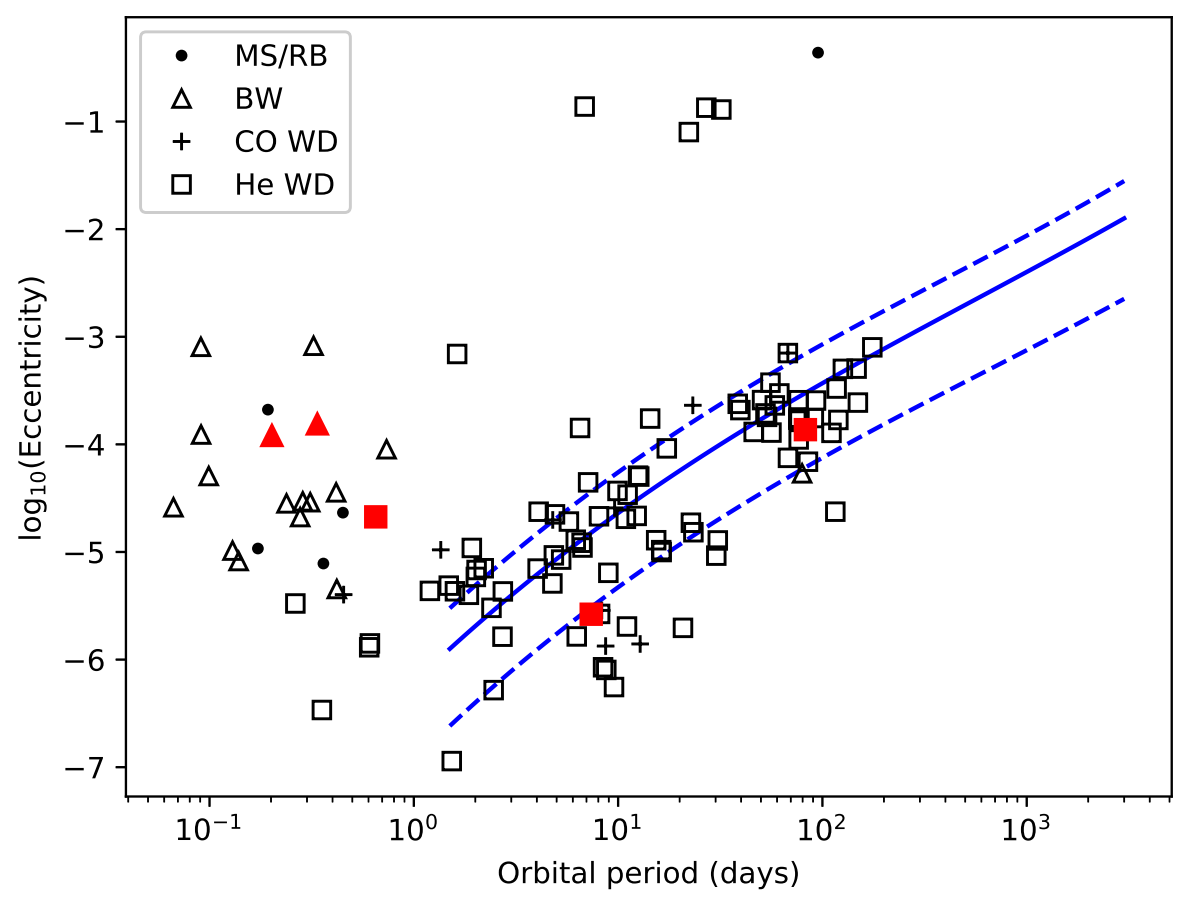

Figure 8. Curves show the theoretical relation between orbital period and eccentricity for NS-WD binaries (Phinney 1992, Phinney \& Kulkarni 1994). The solid curve corresponds to median values and the dashed curves correspond to $95 \%$ confidence contours. Black symbols show field binary MSPs from the ATNF pulsar catalog as follows: redbacks or other systems with a main sequence companion (dots), black widows (open triangles), CO WD companion (crosses), and He WD companion (open squares). Red filled symbols show MSPs from this work with non-zero eccentricities in their best-fit timing solutions. Red filled squares denote the MSPs with He WD companions (J1625-0021, J1824+1014, J2006+0148), and red filled triangles denote the black widows J0251+2606 and J1805+0615.

We have presented the detection of gamma-ray pulsations from eight binary MSPs found in radio searches of Fermi-LAT unidentified sources, 11-year timing solutions based on radio and gamma-ray data for seven of the MSPs, and an updated radio timing solution for the remaining pulsar. Our investigation of a potential correlation between the presence of gamma-ray emission and a short orbital period revealed that selection effects are prominent enough that they cannot be ruled out as the main cause. Based on this analysis we also conclude that the chance of new discoveries in both new and existing pulsar search data can be improved if observations longer than 5-10 minutes are processed using jerk search codes and/or in chunks of that duration in order to minimize the degradation of search sensitivity due to unaccounted for lineof-sight acceleration. The orbital periods and inferred minimum companion masses of our eight MSPs indicate that three are NS-WD binaries, three are black widows with companions whose mass is on the order or $0.02-0.03 \mathrm{M}_{\odot}$, and one is a redback with a companion whose mass is $\gtrsim 0.3 \mathrm{M}_{\odot}$. The remaining MSP $(\mathrm{J} 1908+2105)$ exhibits heavy radio eclipses reminiscent of redbacks but has a minimum companion mass of 
$0.055 \mathrm{M}_{\odot}$, which would be either unusually high for a black widow or unusually low for a redback. This system may represent a rare middle-ground case between these two observational classes.

We thank David Nice for providing a numerical representation of the curves describing the relation between orbital period and eccentricity in Figure 8. We also thank David Smith for advice about working with Fermi-LAT data.

The Arecibo Observatory is operated by the University of Central Florida, Ana G. Mendez-Universidad Metropolitana, and Yang Enterprises under a cooperative agreement with the National Science Foundation (NSF; AST-1744119).

The Fermi-LAT Collaboration acknowledges generous ongoing support from a number of agencies and institutes that have supported both the development and the operation of the LAT as well as scientific data analysis. These include the National Aeronautics and Space Administration and the Department of Energy in the United States, the Commissariat à l'Energie Atomique and the Centre National de la Recherche Scientifique / Institut National de Physique Nucléaire et de Physique des Particules in France, the Agenzia Spaziale Italiana and the Istituto Nazionale di Fisica Nucleare in Italy, the Ministry of Education, Culture, Sports, Science and Technology (MEXT), High Energy Accelerator Research Organization (KEK) and Japan Aerospace Exploration Agency (JAXA) in Japan, and the K. A. Wallenberg Foundation, the Swedish Research Council and the Swedish National Space Board in Sweden. Additional support for science analysis during the operations phase is gratefully acknowledged from the Istituto Nazionale di Astrofisica in Italy and the Centre National d'Études Spatiales in France. This work performed in part under DOE Contract DE-AC02-76SF00515.

Work at NRL was supported by the NASA Fermi program.

Software: PRESTO ${ }^{6}$ (Ransom et al. 2002), TEMPO ${ }^{7}$, PINT $^{8}$ (Luo et al. 2020) Dracula ${ }^{9}$ (Freire \& Ridolfi 2018)

\footnotetext{
${ }^{6}$ https://www.cv.nrao.edu/ sransom/presto

${ }^{7}$ http://tempo.sourceforge.net

8 https://github.com/nanograv/PINT

${ }^{9}$ https://github.com/pfreire163/Dracula
} 


\section{REFERENCES}

Abdo, A. A., Ackermann, M., Ajello, M. et al. 2009, Science, 325, 840

Abdo, A. A., Ackermann, M., Ajello, M. et al. 2009, Science, 325, 848

Abdo, A. A., Ajello, M., Allafort, A. et al. 2013, ApJS, 208, 17

Abdollahi S., Acero F., Ackermann M., Ajello M. et al., 2020, ApJS, 247, 33

Arons, J. 1996, A\&AS, 120, C49

Atwood, W. B., Abdo, A. A., Ackermann, M. et al. 2009, 697, 1071

Atwood, W., Albert, A., Baldini, L. et al. 2013, eConf C121028, 8, in Proc. 4th Fermi Symposium, Monterey

Bruel, P., Burnett, T. H., Digel, S. W. et al. 2018, arXiv:1810.11394

Camilo, F., Kerr, M., Ray, P. S. et al. 2015, ApJ, 810, 85

Chen, H.-L., Chen, X., Tauris, T. M. \& Han, Z. 2013, ApJ, 775, 27

Clark, C. J., Nieder, L., Voisin, G. et al. 2020, arXiv:2007.14849

Cognard, I., Guillemot, L., Johnson, T. J. et al. 2011, ApJ, 732, 47

Cromartie, H. T., Camilo, F., Kerr, M. et al. 2016, ApJ, 819, 34

Crowter, K., Stairs, I. H., McPhee, C. A. et al. 2020, MNRAS, 495, 3052

Deneva, J. S., Ray, P. S., Camilo, F. et al. 2016, ApJ, 823, 105

DuPlain, R., Ransom, S., Demorest, P. et al. 2008, Proc. of SPIE, 7019, 70191D

Foreman-Mackey, D., Hogg, D. W., Lang, D., \& Goodman, J. 2013, PASP, 125, 306

Freire, P. C., Kramer, M. \& Lyne, A. G. 2001, MNRAS, 322, 885

Freire, P. C. C., \& Ridolfi, A. 2018, MNRAS, 476, 4794

Keith, M. J., Johnston, S., Ray, P. S. et al. 2011, MNRAS, 414, 1292
Kerr, M. 2011, ApJ, 732, 38

Luo, J., Ransom, S. M., Demorest, P. et al. 2020, ApJ, submitted

Nieder, L., Allen, B., Clark, C. J. \& Pletsch, H. J. 2020, arXiv:2004.11740

Saz Parkinson, P. M., Dormody, M., Ziegler, M. et al. 2010, ApJ, 725, 571

Phinney, E. S. 1992, Phil.Trans.R.Soc.Lond.A, 341, 39

Phinney, E. S. \& Kulkarni, S. R. 1994, ARA\&A, 32, 591

Pletsch, H. J., Guillemot, L., Allen, B. et al. 2010, ApJ, 744, 105

Pletsch, H. J., Guillemot, L., Fehrmann, H. et al. 2012, Science, 338, 1314

Polzin, E. J., Breton, R. P., Clarke, A. O. et al. 2018, MNRAS, 476, 1968

Polzin, E. J., Breton, R. P., Stappers, B. W. et al. 2019, MNRAS, 490, 889

Ransom, S. M., Eikenberry, S. S. \& Middleditch, J. 2002, AJ, 124, 1788

Ransom S. M., et al., 2011, ApJL, 727, L16

Ray, P. S., Kerr, M., Parent, D., et al. 2011, ApJS, 194, 17

Ray, P. S., Abdo, A. A., Parent, D., et al. 2012, in 2011 Fermi Symposium proceedings - eConf C110509, arXiv:1205.3089

Ray, P. S., Guillot, S., Ransom, S. M. et al. 2019, ApJ, 878L, 22

Strader, J., Swihart, S.; Chomiuk, L. et al. 2019, ApJ, 872, 42

Tabassum, S., Roberts, M., Ransom, S. et al. 2020, AAS, 235, 302.17

Tauris, T. M. \& Savonije, G. J. 1999, A\&A, 350, 928

Yao, J. M., Manchester, R. N. \& Wang, N. 2017, ApJ, 835, 29

Zharikov, S., Kirichenko, A, Zyuzin, D. et al. 2019, MNRAS, 489, 5547 